\title{
Potential Clinical Applications of PET/MR Imaging in Neurodegenerative Diseases
}

\author{
Alexander Drzezga ${ }^{1}$, Henryk Barthel $^{2}$, Satoshi Minoshima*3, and Osama Sabri*2 \\ ${ }^{I}$ Department of Nuclear Medicine, University of Cologne, Cologne, Germany; ${ }^{2}$ Department of Nuclear Medicine, University of \\ Leipzig, Leipzig, Germany; ${ }^{3}$ Division of Nuclear Medicine and Radiology, University of Washington Medical Center, Seattle, WA
}

\begin{abstract}
Neurodegenerative disorders such as Alzheimer disease are among today's most alarming health problems in our aging society. The clinical assessment of neurodegenerative disorders benefits from recent innovations in the field of imaging technology. These innovations include emerging tracers for molecular imaging of neurodegenerative pathology and the introduction of novel integrated PET/MR imaging instruments. Because both PET and MR imaging procedures have shown critical value in the diagnostic work-up of neurodegenerative disorders, the combination of both imaging modalities in the form of an integrated PET/MR imaging system may be of value. This combination includes practical methodologic advantages and an improved workflow facilitated by the combined acquisition of dual-modality data. It offers clinical advantages because of the systematic combination of complementary information, potentially allowing the creation of novel integrated imaging biomarkers. The effectiveness of new disease-modifying treatments may depend on the timely initiation of therapy before irreversible neuronal damage in slowly progressive neurodegenerative disorders. Integrated $\mathrm{PET} / \mathrm{MR}$ imaging may be able to improve such early diagnosis through both structural and functional information.
\end{abstract}

Key Words: hybrid imaging; PET/MR; MR/PET; neurodegeneration; dementia

J Nucl Med 2014; 55:47S-55S

DOI: 10.2967/jnumed.113.129254

$\mathbf{T}$ he term neurodegeneration includes several disorders that are characterized by a progressive dysfunction and, ultimately, the loss of neurons. The most frequent forms include cognitive disorders such as Alzheimer disease and other forms of dementia, as well as movement disorders such as Parkinson and Huntington disease. It is estimated that more than 5 million people have Alzheimer disease, the most frequent form of neurodegeneration in the United States. The number is expected to increase to 14 million by $2050(1,2)$. In recent years, growing evidence suggests that, despite different initial clinical manifestations, overlapping pathophysiologic processes may be involved in various forms of neurodegenerative disorders (3). Consistently, increased

\footnotetext{
Received Mar. 21, 2014; revision accepted Apr. 23, 2014.

For correspondence or reprints contact: Alexander Drzezga, Department of Nuclear Medicine, University Hospital of Cologne, Kerpener Strasse 62, 50937 Cologne, Germany

E-mail: alexander.drzezga@uk-koeln.de

${ }^{*}$ Contributed equally to this work.

Published online May 12, 2014.

COPYRIGHT (c) 2014 by the Society of Nuclear Medicine and Molecular Imaging, Inc.
}

production, misfolding, and pathologic aggregation of specific peptides have been identified in many neurodegenerative disorders, representing an early and potentially causal phenomenon. Such phenomena include, for example, $\beta$-amyloid plaques and $\tau$-protein aggregates in Alzheimer disease, as well as other forms of aggregates such as the $\alpha$-synuclein aggregates (Lewy bodies) found in Parkinson disease and dementia with Lewy bodies. Common disease models suggest that these pathologies contribute to the development of other characteristic features of neurodegeneration such as neuronal dysfunction and neuronal loss in the central nervous system (4), which, again, can be found consistently in different forms of neurodegeneration.

Numerous studies confirm that the different aspects of this neuropathologic cascade can be successfully captured in vivo using suitable neuroimaging methods-in particular, MR imaging and PET. For example, structural MR imaging allows the measurement of neuronal loss (atrophy) (5); ${ }^{18} \mathrm{~F}$-FDG PET allows the assessment of neuronal dysfunction $(6,7)$; and several newer radiotracers ( ${ }^{18} \mathrm{~F}$-florbetapir, ${ }^{18} \mathrm{~F}$-florbetaben, ${ }^{18} \mathrm{~F}$-flutemetamol) allow imaging of cerebral amyloid deposits $(8-10)$. Some of these markers have already found their way into clinical application; others have proven high value in the scientific evaluation of disease mechanisms. Recent biomarker studies in familial forms of dementia indicate complementary roles of different imaging biomarkers at different disease stages $(3,11,12)$.

Both improved diagnosis and better understanding of the disease processes are relevant with respect to the numbers of patients affected by these disorders and their impact on society. It is estimated that more than 5 million people currently have Alzheimer disease, and the number will increase to 14 million in $2050(1,2)$. Therapeutic options are limited to mild symptomatic improvements. However, early diagnosis and more accurate differential diagnoses may offer greater benefits to the patients if appropriate therapies can be instituted before substantial neuronal damage occurs in slow, progressive neurodegenerative disorders.

Regarding the value of neuroimaging markers, it has been shown that the combination of PET and MR imaging may offer various methodologic advantages and potentially lead to improved diagnostic classification, disease staging, and prognostic evaluation in addition to advancing a better understanding of interrelated disease pathomechanisms. Integrated PET/MR technology (13) may be ideally suited for scientific and clinical assessment of neurodegenerative disorders. In addition to systems combining the two modalities in a single room (e.g., Philips Ingenuity), fully integrated solutions have recently been introduced. The first prototype of an integrated PET/MR scanner consisted of an MR imagingcompatible brain PET insert positioned inside a commercially available 3-T MR imaging Trio system (Siemens Healthcare) (14). In 
2010, a fully integrated PET/MR scanner also became available for human whole-body imaging (Siemens Biograph mMR) (15), and other industries (e.g., GE Healthcare) are currently working on a similar concept.

Although the integration of brain PET and MR imaging information has long been performed for clinical and scientific purposes using independently acquired datasets, the simultaneous acquisition of PET/MR imaging may create unique opportunities that are not possible with separate acquisitions. Such opportunities include methodologic and clinical advantages as discussed in the following sections.

\section{METHODOLOGIC ADVANTAGES}

The combination of PET and MR imaging information, particularly when acquired at the same time, offers several potential methodologic advantages in neuroscience applications. In addition to the combination of complementary information, the imaging data of one modality may be used to improve the quality and interpretation of the other modality.

\section{Systematic Addition of Anatomic Information}

Anatomic information that can be derived from PET imaging is relatively limited, due in part to the limited resolution of PET. However, even more important, brain uptake of specific PET tracers allows visualization only of specific structures (e.g., the basal ganglia) or pathologies (e.g., inflammation, tumor tissue). Moreover, resulting distribution patterns may be fundamentally different between patients and healthy subjects, showing more uptake (e.g., amyloid imaging) or less uptake (e.g., ${ }^{18}$ F-FDG PET in dementia or dopamine-receptor PET in Parkinson disease). Consequently, the exact anatomic localization of pathologic findings and regional comparison between patient and control data can be hampered by lack of anatomic information on PET findings alone. Also, regional comparison between baseline and follow-up information in patients (e.g., during therapy) in corresponding regions may be difficult when patterns of tracer uptake change. The systematic addition of high-resolution MR imaging information to PET data provides accurate and consistent information on underlying structures, helps overcome difficulties in anatomic localization on PET images, and improves scan interpretation. Historically, this has been done by coregistraton of independent PET and MR imaging datasets after data acquisition (16). Definition of regions of interest on the basis of accurately coregistered MR data may significantly improve the reliability and consistency of the quantitative evaluation of PET data. This benefit is particularly relevant in situations in which the PET tracers specifically bind to subcortical or smaller brain structures, that is, without widespread uptake in larger cortical brain areas, allowing straightforward image data coregistration of separately acquired PET and MR data (Fig. 1).

Additionally, anatomic abnormalities such as arachnoidal cysts and certain pathologies (e.g., older hemorrhages), which can be detected by means of MR imaging, may influence the tracer uptake pattern (e.g., causing circumscribed tracer defects). For example, vascular lesions may lead to reduced uptake in the basal ganglia, mimicking nigrostriatal neurodegeneration. Such abnormalities may accompany other disorders of the brain, and knowledge of their presence may lead to a more confident interpretation of the PET data.

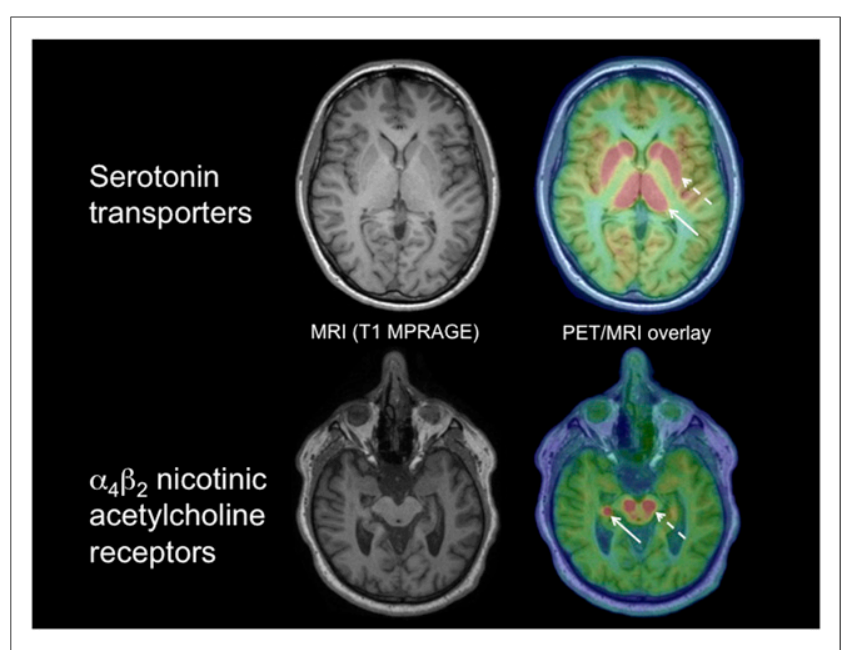

FIGURE 1. Serotonin transporters (SERT) were imaged in dementia patient with suspected depressive comorbidity using ${ }^{11} \mathrm{C}-\mathrm{DASB}$, and a4ß2 nicotinic acetylcholine receptors using ${ }^{18} \mathrm{~F}-\mathrm{A} 85380$ in Parkinson disease patient with suspected dementia comorbidity. Dashed and full arrows in SERT image represent striatum and thalamus; those in nAChR image, substantia nigra and lateral geniculate nucleus. Note anatomic assignment of focal PET tracer uptake. Images were acquired at Leipzig University, Germany, on integrated Siemens mMR system (funded by Deutsche Forschungsgemeinschaft, Großgeräteinitiative).

\section{Correction for Atrophy and Partial-Volume Effects}

Tracer uptake in the brain measured by PET is influenced by the underlying brain structure- that is, the distribution and volume of gray and white matter. Spill-over effects may lead to over- or underestimation of the actual regional PET tracer uptake in a given brain region, depending on the size of the structure and the intensity and distance of uptake in neighboring regions. Particularly in conditions associated with cerebral atrophy, such as neurodegenerative disorders, it may be vital for the quantification of observed PET findings to integrate the underlying structural framework obtained by MR imaging into the interpretation (see the following text). Misinterpretation because of cortical atrophy may lead to under- or overestimation of the pathologic findings, depending on the tracer used.

In situations in which reduced PET tracer uptake is a sign of pathology, such as ${ }^{18} \mathrm{~F}-\mathrm{FDG}$ imaging in Alzheimer disease or dopamine transporter imaging in Parkinson disease ("cold spot" imaging), brain atrophy might lead to a false-positive PET diagnosis. For "hot spot" imaging, on the other hand, as in amyloid PET in Alzheimer disease, accompanying atrophy works against the PET signal and potentially decreases the diagnostic power of PET. Therefore, it is desirable to develop and implement effective correction of brain atrophy and partial-volume effects into clinical routine.

The integration of atrophy into PET findings may be performed by individual visual assessment, by segmenting the gray matter of individual MR imaging data (8), but also by application of advanced partial-volume effect correction algorithms. Again, exact anatomic coregistration of MR imaging and PET data is a requirement for such approaches. Integrated PET/MR imaging offers the opportunity to perform atrophy correction of the PET data on a systematic basis using consistent MR information (17) and to implement partial-volume effect correction in a clinical routine imaging setting. 


\section{Correction of Factors That Influence Regional Tracer Supply}

The regional brain distribution of PET tracers can be influenced by several physiologic and pathophysiologic factors - for example, regional perfusion or the integrity of the blood-brain barrier. Regional inhomogeneity of these factors may occur, for example, in cerebrovascular disease, stroke, or cerebral ischemia, when regional hypoperfusion may result in decreased tracer supply but also delayed tracer washout. For example, in scientific studies of receptor displacement, specific tasks or conditions may alter not only regional transmitter release but also blood flow and thus may affect the resulting tracer distribution pattern. Correction of tracer uptake for these factors is performed by means of tracer kinetic modeling. In particular, if no suitable reference region can be defined, this approach usually requires an arterial input function, which must be performed invasively by puncturing a peripheral artery. Thus, truly quantitative evaluation is often limited to research applications and too rarely has found its way into clinical neuroimaging studies.

The simultaneous acquisition of MR data may allow the gathering of information on several of the previously mentioned cofactors and thus make it possible to correct the PET data accordingly and allow a realistic estimation of the true target concentration. For example, MR procedures using injection of contrast medium allow the assessment of different parameters such as cerebral blood flow, blood volume, permeability, and blood-brain barrier disruption. Perfusion can also be assessed without contrast material by methods such as arterial spin labeling (ASL). The high temporal resolution and lack of radiation exposure of MR imaging may allow monitoring changes in regional blood flow not only at the initial peak of injection but also throughout the examination (e.g., using functional MR imaging and blood oxygen leveldependent contrast) and modeling PET tracer distribution quantitatively. The definition of vascular regions of interest on the basis of MR information (e.g., carotid arteries) may be used to measure the vascular PET signal and thus to obtain reliable tracer input function. One approach in this regard is to use time-of-flight MR angiography (18). Finally, by combining MR imaging- and PET-based information, it may be possible to replace the need for an invasive arterial input function; however, research is continuing $(19,20)$.

\section{Quantification of PET Tracer Uptake}

In situations in which quantitative information as to the PET tracer uptake in the brain is relevant-for example, follow-up evaluation in therapy monitoring - integrated PET/MR imaging must be validated for its capability as a quantitative imaging tool. The shortcomings of suboptimal attenuation correction of PET emission data caused by inaccurate assumption of bone attenuation measured using the 2-point (fat, water) Dixon-based attenuation correction approach (21)—which generates attenuation coefficient maps solely for air, lung, soft tissue, and fat using MR imagingmust be overcome. If quantitative accuracy improves, integrated PET/MR imaging might even improve quantification of PET tracer uptake as compared with stand-alone PET or PET/CT data-for example, by correction for the patient's lean body mass.

\section{Head Motion Correction}

In patients with neurologic disorders such as dementia or Parkinson disease, head motion during the acquisition of the PET data is a significant issue leading to impaired image quality. For simultaneous PET/MR imaging acquisition, it has been shown that it may be possible, first, to register patient head movement during the entire PET duration by continuous MR monitoring of head motion and, second, to subsequently correct PET data for the motion (22). Several frames are often acquired in brain imaging procedures of patients with neurodegeneration to allow the selection of at least one frame that is not affected by major head movement. Alternatively, in list mode acquisition, segments of the scan that are less affected by motion can be isolated and used for reconstruction. Of course, these approaches result in heterogeneous data quality because of the resulting variable acquisition times. In contrast, accurate simultaneous motion correction would potentially allow obtaining high-quality image data in moving patients in a shorter acquisition time with consistent counting rate and statistics.

\section{Cross-Validation of Imaging Procedures}

Simultaneous data acquisition by MR imaging and PET offers a unique opportunity to cross-validate new and existing imaging methods. In particular, the option to provide absolutely quantitative information with PET-for example, on cerebral blood flow or metabolism - could be used to validate or scale MR tools such as ASL, which may provide similar information (23). The high value of ${ }^{18}$ F-FDG in the early and differential diagnosis of dementia has not been reproduced with, for example, ASL MR imaging methods. However, PET/MR imaging may assist in further improving and validating these techniques in a true 1:1 comparison under identical conditions. An option to combine ASL with amyloid PET in a single PET/MR imaging session may offer an opportunity to answer questions on molecular pathology and neuronal dysfunction at the same time (see "Conclusion").

\section{Methodologic Limitations}

Despite enthusiasm for integrated PET/MR imaging, several methodologic limitations and new methodologic challenges have arisen from the integration of PET and MR in a single instrument, which required substantial changes in technical configuration, particularly of the PET component. To operate in a strong magnetic field, conventional photomultipliers had to be exchanged for avalanche photodiodes, the scanner geometry had to be adapted, and PET data must be acquired in the presence of an MR head coil. Most importantly, no CT data for attenuation correction are available, so attenuation maps must be generated on the basis of MR information. The 2-point Dixon approach does not allow a direct assessment of the specific contribution of bone to photon attenuation. Recent studies have shown that these technical peculiarities may lead to relevant differences in the PET data of PET/MR imaging as compared with PET/CT data, particularly in the brain $(24,25)$. In this context, further improvement of the methodology is certainly required.

\section{DIAGNOSTIC IMPROVEMENT}

The diagnosis of neurodegenerative disorders may be significantly improved by systematically combining the complementary information obtained by PET and by MR imaging.

Imaging in neurodegenerative dementia may be a particularly well-suited example of the potential value of systematic coacquisition of PET and MR data. A definite diagnosis of the type of dementia has thus far been possible only by histopathologic postmortem assessment of brain tissue. Clinical diagnosis on the basis of neuropsychologic and symptomatic evaluation has several shortcomings: First, it is now well accepted that pathologic changes in the brain start to appear years to decades before symptoms $(26,27)$. This fact is hampering the early diagnosis of ongoing 
neurodegeneration and, consequently, early treatment interventions or therapy trials in a stage before irreversible neuronal damage.

Additionally, considerable symptomatic overlap has been shown between different forms of dementia, which leads to clinical misclassification regarding the causal pathology underlying the symptomatic appearance. Finally, disease progression may not be well mirrored in the degree of cognitive impairment, which is subject to distinct variation. The assessment of clinical symptoms may therefore not represent an ideal tool for followup and therapy monitoring in dementia. The example of dementia illustrates that neurodegenerative disorders in general may not be well characterized on the basis of their symptomatic appearance only. This insight has also found its way into recent diagnostic criteria-for example, in Alzheimer disease. These criteria now consider the disorder to be a disease continuum, including a presymptomatic phase, a phase of mild unspecific cognitive symptoms, and a phase of clinically manifest dementia $(28,29)$. Similar concepts apply to most other forms of neurodegeneration. This dissociation between symptomatic appearance and neuropathology emphasizes the need for suitable biomarkers. Consequently, the more recent diagnostic concepts explicitly advocate the use of biomarkers - including imaging - in the diagnostic evaluation of the stages of neurodegenerative disease $(28,29)$. In the following paragraphs, we try to elucidate the individual value of typical dementia neuroimaging biomarkers before discussing their potential complementary combination.

\section{Individual Value of Neuroimaging Markers of Neurodegeneration}

MR Imaging. Specific patterns of cerebral atrophy can be detected using structural MR imaging for different forms of neurodegenerative dementias $(30,31)$. Most clearly, these findings were shown by means of statistical groupwise comparison of patients versus healthy control subjects. However, the individual value of the specific patterns of atrophy for early diagnosis, prognosis, and differential diagnosis is less apparent (32-35). A potential reason for this can be found in the assumption that structural and atrophic changes in the brain are a downstream phenomenon in the process of neurodegeneration (36). Furthermore, significant changes of brain volume have been described for healthy aging as well (37) and may interfere with the detection of specific pathologic changes. However, studies were able to show that longitudinal loss of brain substance (i.e., rate of atrophy) over a relatively short time (e.g., 1 y), may be highly predictive for future cognitive decline (38). In addition to detection of characteristic patterns of atrophy, MR imaging is the ideal tool for the exclusion of nonneurodegenerative and potentially treatable causes for cognitive symptoms - that is, brain tumors and vascular or inflammatory abnormalities. For this reason, morphologic imaging procedures such as CT or, preferably, MR imaging of the brain are suggested as an indispensable part of the dementia work-up (39).

${ }^{18} F$-FDG PET. PET imaging procedures have been shown to have a high value as a biomarker for reliable diagnosis and differential diagnosis of dementing disorders and even for prediction in predementia stages, such as mild cognitive impairment. This statement refers in particular to ${ }^{18} \mathrm{~F}$-FDG PET, which is a well-established tool to measure regional glucose metabolism indicating neuronal function. In different forms of neurodegenerative dementias, specific patterns of neuronal dysfunction have been described (40). The value of these patterns for the reliable diagnosis of Alzheimer disease in the individual has often been shown, even by postmortem autopsy $(41,42)$. Many studies were able to show characteristic abnormalities of cerebral metabolism in mild cognitive impairment, which is valuable for the prediction of Alzheimer disease, and it has been reported that these abnormalities are of higher diagnostic value than those shown on structural MR imaging (43).

Amyloid Imaging. More recently, PET tracers for molecular imaging of $\beta$-amyloid plaques, a neuropathologic hallmark of Alzheimer disease, have been introduced $(8,44-46)$.

This type of imaging tool may allow founding the diagnosis of a neurodegenerative disorder on the underlying pathology rather than on the symptomatic appearance. It has been reported repeatedly that significantly increased amyloid tracer uptake can be documented, even in a relevant percentage (20\%-30\%) of healthy elderly subjects, possibly reflecting ongoing neuropathology in asymptomatic stages (47-49). A predictive value of an amyloid positive finding in these subjects with regard to later cognitive decline has been discussed (50-52).

These findings indicate that amyloid imaging may be useful for the detection of Alzheimer disease pathology, potentially even earlier than with ${ }^{18} \mathrm{~F}$-FDG PET. Particularly with regard to new therapy options directed toward amyloid pathology, amyloid imaging may be a valuable tool to select patients with proven amyloid pathology in the early stages of disease. Recent antiamyloid trials investigating monoclonal antibody therapies in patients with early Alzheimer disease did not successfully reach their predefined endpoints (53). These types of causal therapy strategies would have to be initiated in an earlier phase of disease, that is, before irreversible neuronal damage. However, amyloid imaging showed that up to $36 \%$ of the subjects included in these trials did not fulfill imaging criteria for cortical amyloid deposition (elevated neocortical tracer uptake as compared with the cerebellar reference region). Consequently, amyloid imaging may provide earlier and more reliable proof of the target pathology for these trials.

Regarding differential diagnosis, amyloid imaging may also bear specific potential. Although amyloid deposits are not exclusively found in Alzheimer disease but, for example, also in dementia with Lewy bodies (54), evaluation of the presence of amyloid pathology may be relevant in the diagnostic distinction between the various etiologic forms of neurodegeneration. Amyloid imaging may allow the classification of atypical forms of Alzheimer disease such as posterior cortical atrophy or the logopenic variant of progressive aphasia. Amyloid imaging has also been shown to be useful for the differentiation between amyloidnegative frontotemporal dementia and Alzheimer disease $(55,56)$. Furthermore, amyloid imaging may be helpful for objective longitudinal therapy monitoring. Although a major reduction of the cerebral amyloid load may not be expected even during successful treatment, a stagnation of further amyloid deposition as compared with a placebo group appears possible (57).

\section{Multimodal Approaches}

Not only did the imaging biomarkers discussed previously find their way into the diagnostic criteria, but also their complementary value with regard to the diagnostic information is now well accepted. In particular, results from recent studies on familial Alzheimer disease support this notion (12). These studies indicate that imaging markers of amyloid deposition and of neuronal dysfunction and loss may all become positive before manifest cognitive impairment but in a sequential order, with amyloid markers 


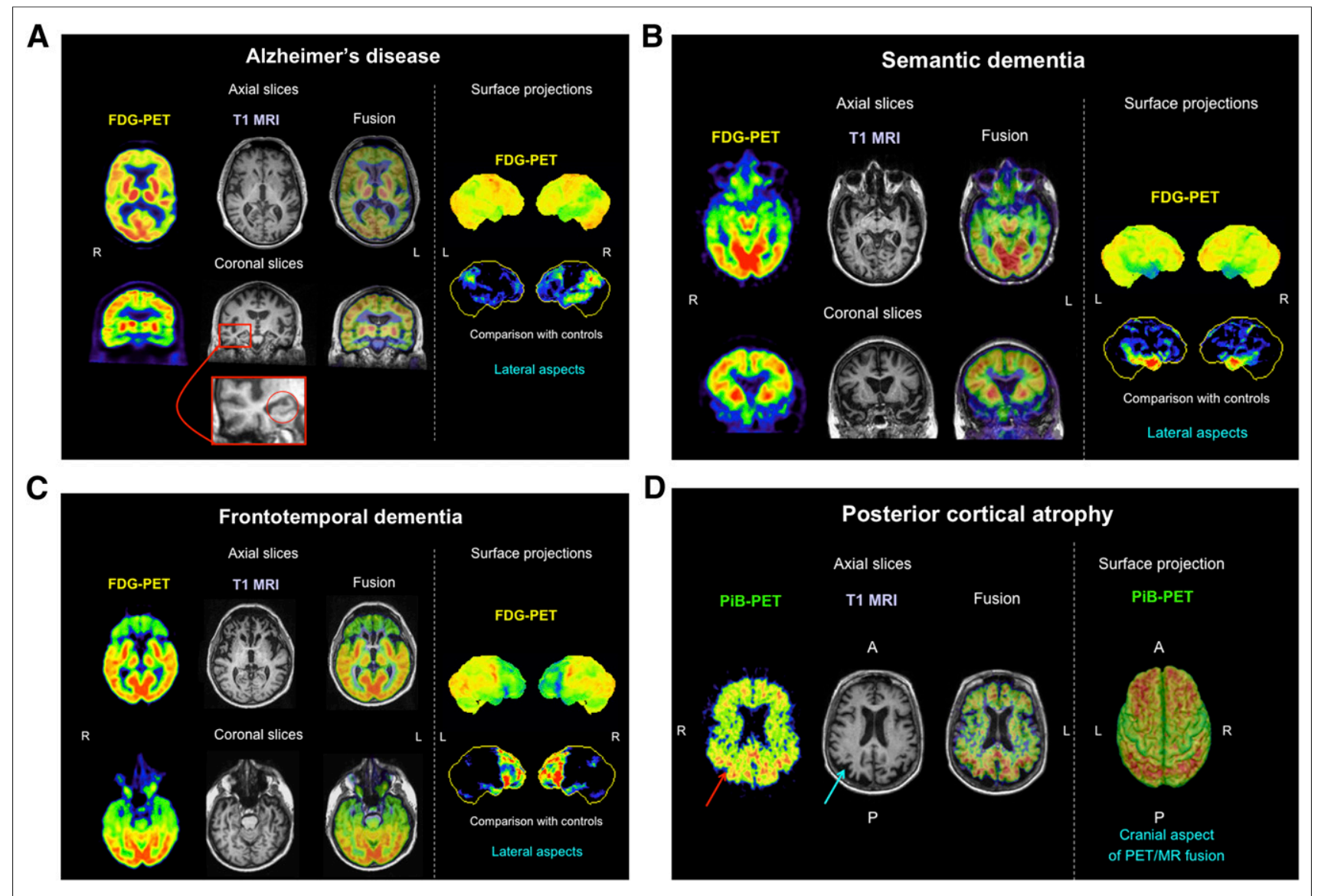

FIGURE 2. Multimodal differential diagnosis of dementia. (A) Typical patient with Alzheimer disease. Hypometabolism in posterior temporoparietal cortical regions was identified on ${ }^{18} \mathrm{~F}-\mathrm{FDG}$ PET scan as well as typical pattern of cortical atrophy, including mesial temporal lobe (red box). (B) Typical patient with semantic dementia. Hypometabolism in bilateral temporal cortical regions was detected on ${ }^{18} \mathrm{~F}-\mathrm{FDG}$ PET scan as well as typical pattern of cortical atrophy, which is pronounced in temporal polar regions. (C) Typical patient with frontotemporal dementia. Hypometabolism and corresponding atrophy were identified in frontal cortex and in bilateral temporal cortical regions. (D) Example of posterior cortical atrophy. In this example, major atrophy was observed in posterior portions of brain on structural MR imaging data obtained on PET/MR scanner (blue arrow). Also, ${ }^{11} \mathrm{C}$-Pittsburgh compound B (PiB) amyloid scan has been performed in this patient. In combination with MR imaging data, it is obvious that posterior portions of brain express distinct amyloid load despite massive atrophy (red arrow). These findings support diagnosis of posterior cortical atrophy, which is assumed to be based on Alzheimer-type pathology. All images in Figure 2 were acquired at Technische Universität München, Munich, Germany, on integrated Siemens mMR system (funded by Deutsche Forschungsgemeinschaft, Großgeräteinitiative).

turning positive many years before dementia, followed by neuronal dysfunction and, finally, structural loss. Furthermore, these imaging biomarkers appear to show a nonparallel course of change over time in relation to the progressive decrease of cognitive impairment. Whereas amyloid deposition appears to increase steeply at first, it then seems to plateau as soon as manifest dementia is present; it does not show linear correlation with further cognitive decrease thereafter. On the other hand, structural imaging and volumetry initially show relatively minor changes becoming an exponential progression with the onset of cognitive decline. Imaging of neuronal function seems to show a relatively linear progression over time in correlation with a decrease in cognitive function. However, also with ${ }^{18}$ F-FDG PET, effects of cognitive reserve have been shown to modulate the relation between the extent of imaging abnormality and the degree of cognitive impairment (58).

These insights have several consequences with regard to the value of multimodal imaging. Examples are listed in the following section.
Definite Diagnosis. Regarding the complementary value of the discussed imaging markers for detecting different forms of neuropathology, the combination of these tools may aid in accumulating evidence for a specific neurodegenerative disorder. This assumption is already part of recent diagnostic criteria for Alzheimer disease, which postulate that a combination of several positive biomarkers (amyloid pathology and neuronal injury) increases the likelihood that the patient has the disease $(28,59)$. Indeed studies have shown, for example, that combinations of structural MR imaging and ${ }^{18}$ F-FDG PET or MR imaging and amyloid imaging revealed higher diagnostic accuracy as compared with either single method $(33,60)$.

Early Diagnosis. Regarding the expected time course of the mentioned biomarkers, amyloid imaging in principle may allow early detection of ongoing amyloid pathology even in asymptomatic stages, but to date, no information is available on the expected time to conversion. Regarding the prediction of Alzheimer disease in the stage of mild cognitive impairment, amyloid PET and ${ }^{18} \mathrm{~F}-$ FDG PET both appear to be superior to structural MR imaging 
and to have similar overall levels of predictive accuracy (61-63). Whereas amyloid PET appears to be somewhat more sensitive, ${ }^{18}$ F-FDG PET seems to show higher specificity and greater short-term predictive value (64). Structural MR imaging may permit a better estimation of time to conversion in amyloid-positive subjects than the amyloid level itself (65). These results indicate that depending on the diagnostic question, combinations of these biomarkers may have relevant added value in early diagnosis. Correspondingly, the combination of amyloid imaging and a marker of neuronal injury (e.g., a simultaneous amyloid PET and structural MR imaging scan) is considered to increase the probability that a patient has Alzheimer disease in preclinical and mildly cognitively impaired stages of disease, according to the current guidelines $(29,66)$. Furthermore, the combination of several imaging biomarkers may allow assessment not only of the potential risk but also of the expected time to onset of cognitive problems. In the same context, another recent finding is of particular importance: several studies now indicate that markers of neuronal injury, which are predictive of later cognitive decline, may be found in the presence of negative markers of amyloid pathology in some subjects who are cognitively healthy at the time of the initial examination (67). Consequently, only multimodal imaging approaches that allow the assessment of neuronal injury and amyloid deposition may be able to capture all subjects at risk for neurodegeneration at the earliest possible time.

Differential Diagnosis. Adding MR imaging information to PET information with regard to differential diagnosis of neurodegenerative disorders can be strongly advocated for several reasons. First, the quantification of atrophy may improve image interpretation, as mentioned previously. Furthermore, the combination of specific patterns of brain atrophy in MR imaging with characteristic patterns of neuronal dysfunction in ${ }^{18} \mathrm{~F}-\mathrm{FDG}$ PET $(68)$, or with results from amyloid imaging, may help guide the way to a more confident diagnosis combining causal neuropathology with resulting neurodegenerative patterns. Figure 2 shows examples of combinations of PET and MR imaging data that lead to increased diagnostic certainty by accumulating evidence. Regarding the detection or exclusion of nonneurodegenerative pathologies, MR imaging can be considered a mandatory prerequisite in the differential diagnostic work-up of dementia. However, mixed pathologies may be present in many cases and may only be detected using a multimodal approach. For example, MR imaging can detect vascular abnormalities associated with vascular dementia. Additionally, a large overlap exists between vascular and neurodegenerative pathologies. The verification of vascular abnormalities as copathology of neurodegeneration may allow therapeutic interventions (e.g., regulation of hypertension) and thus may lead to a better outcome. Particularly in older patients, amyloid pathology may be present without representing the main or exclusive course of cognitive impairment. In the older population, the likelihood of multiple cerebral pathologies (e.g., amyloid and vascular lesions) is expected to be high. Figure 3 shows a dementia patient who was positive in amyloid PET. In this case, susceptibility-weighted imaging, as obtained in parallel to the PET information on an integrated PET/MR imaging system, identified additional vascular pathology (microbleeds), suggesting the presence of cerebral amyloid angiopathy.

Follow-Up and Therapy Monitoring. For follow-up evaluation, different combinations of diagnostic modalities may be chosen with PET/MR imaging - for example, for therapy monitoring. Depending on the type of therapy and the stage of treatment

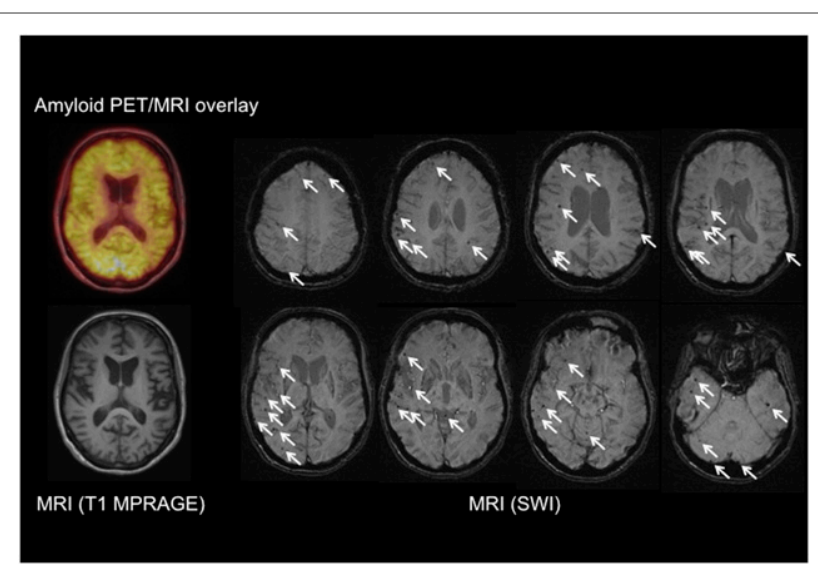

FIGURE 3. Example of PET/MR scan in which ${ }^{18} \mathrm{~F}$-florbetaben PET shows intense gray matter tracer uptake, indicating cortical amyloid load. Simultaneous susceptibility-weighted imaging (SWI) MR image revealed scattered microbleeds. Taken together, these results point to cerebral amyloid angiopathy. Images were acquired at Leipzig University, Germany, on integrated Siemens mMR system.

initiation, it may be of particular interest to quantify the therapeutic impact on the amyloid burden or on neuronal dysfunction in combination with the effect on progression of neuronal loss. Particularly in neurodegenerative disorders, atrophy may lead to under- or overestimation of the pathology in the PET data-for example, the extent of cortical hypometabolism interpreted as a marker of neuronal dysfunction may be overestimated in severe atrophy, whereas the regional amyloid load could be underestimated. These effects may be critical in longitudinal and followup studies. For example, progressive atrophy over time may lead to underestimation of increasing amyloid load in the remaining brain tissue. The combination of PET and MR imaging at baseline and follow-up may allow disentangling these diverging pathologies and help to accurately quantify disease progression or therapy effects over time. As mentioned previously, the systematic combination of structural MR imaging and PET may allow the performance of partial-volume effect correction and the estimation of the true PET tracer uptake per given volume of brain tissue. Also, with regard to the observed different time courses of the various imaging biomarkers, as mentioned previously, multimodal assessment can be expected to allow a much better-informed quantification of disease progress or of therapy effects on several scales.

\section{IMPROVED DIAGNOSTIC WORKFLOW ALGORITHMS}

In principle, using PET/MR imaging, it should be possible in the future to accomplish all brain imaging necessary for the assessment of neurodegenerative disorders in a single visit, saving investigation time and increasing patients' and doctors' comfort. For reasons of efficiency, it will not be possible to perform all available imaging tests for all indications. Thus, an intelligent combination of the neuroimaging biomarkers will be required. Further studies are necessary to identify optimal combinations and protocols for the individual diagnostic problem.

In this context, it may be possible to substitute the information derived from ${ }^{18} \mathrm{~F}$-FDG PET by means of suitable MR sequences or by collecting dynamic (perfusion) data in an amyloid scan $(23,69-$ 71). One example of simultaneous amyloid load and blood flow imaging using integrated PET/MR imaging in a dementia patient 
is given in Figure 4. This practice would allow performing a single PET/MR examination that nevertheless provides information on molecular neuropathology, neuronal dysfunction, and structural atrophy.

Figure 5 shows the potential of such a "one-stop shop" PET/MR imaging algorithm and compares it with the diagnostic tools currently available to support a clinical diagnosis of Alzheimer disease.

\section{SCIENCE}

Regarding scientific applications in the field of neurodegeneration, PET/MR imaging opens a whole array of possibilities. Particularly, the integrated technology that allows simultaneous acquisition of PET and MR imaging data may help to crossevaluate MR imaging methods such as ASL or resting state against ${ }^{18} \mathrm{~F}$-FDG PET with regard to their value in the diagnosis of neurodegenerative disorders.

In addition, the combination of the various available tracers and MR brain imaging procedures may help us understand the regional and temporal cross-relation between different pathologies. Not only the order of appearance but also the quantitative and topographic correlation of pathophysiologic changes can be assessed, potentially leading to the detection of causal relations. Among many potential applications, for example, the combination of MR spectroscopy and specific PET tracers may yield interesting insights into disease pathomechanisms, including changes in neuronal metabolism (e.g., aerobic glycolysis) or turnover of neurotransmitters. Studies of functional MR imaging and structural connectivity (diffusion tensor imaging) combined with PET examinations may allow the study of the distribution and expansion of neurodegenerative pathologies in relation to networks of the brain. Mechanisms and effects of novel therapeutic approaches such as stem cell transplants or neuroprotective agents may be investigated. However, an exhaustive discussion of the scientific opportunities of PET/MR imaging with regard to neurodegeneration is beyond the scope of this article, which is focused on clinical applications.

\section{CONCLUSION}

Taken together, integrated brain PET/MR imaging has great potential to improve and simplify the diagnosis of neurodegener-

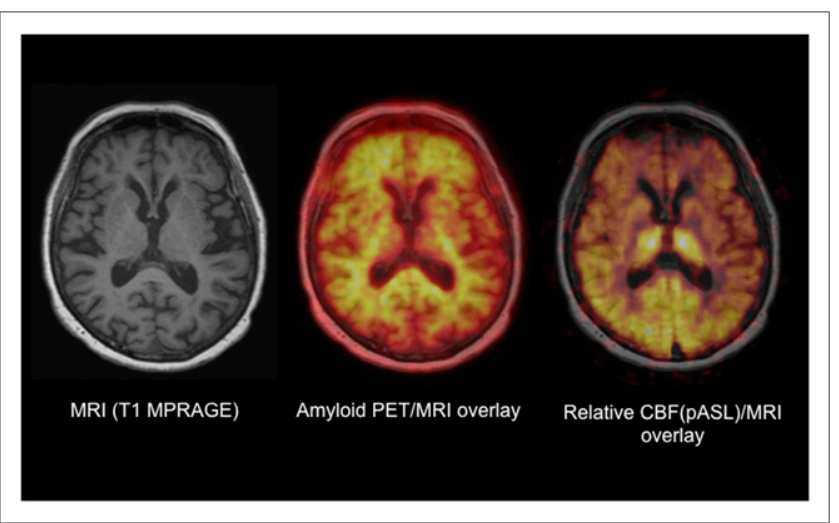

FIGURE 4. Simultaneous measurement of amyloid load and blood flow in dementia. Amyloid load was imaged using ${ }^{18} \mathrm{~F}$-florbetaben PET, and relative cerebral blood flow, by means of pulsed arterial spin labeling (pASL) MR imaging. This "one-stop shop" data acquisition, providing both pathology and neuronal injury biomarker to support clinical diagnosis of Alzheimer disease, takes about $20 \mathrm{~min}$, which increases patient comfort. Images were acquired at Leipzig University, Germany, on integrated Siemens mMR system.

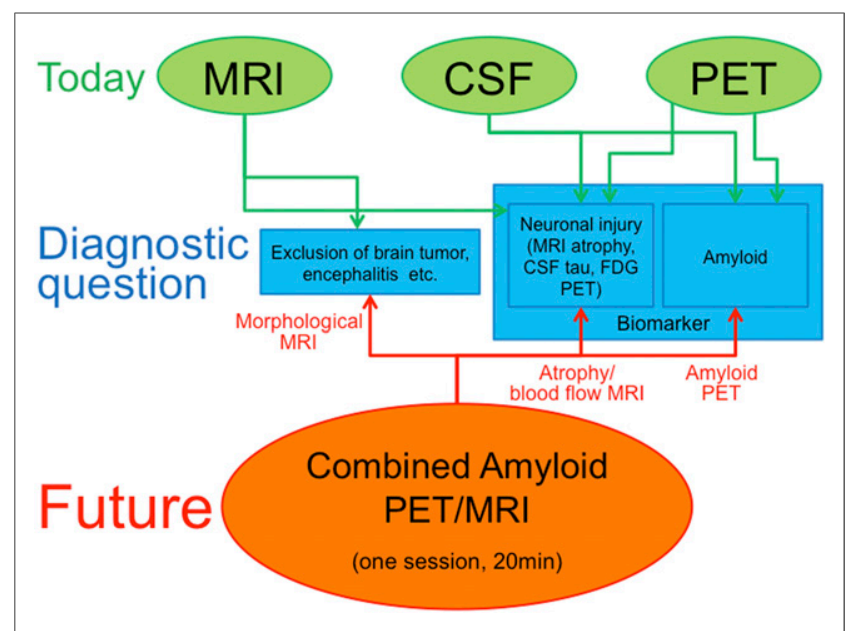

FIGURE 5. Potential advantages of "one-stop shop" integrated brain $\mathrm{PET} / \mathrm{MR}$ algorithm as compared with diagnostic tools currently available to establish diagnosis of Alzheimer disease.

ative disorders because of its several methodologic advantages. These include the systematic integration of anatomic information into interpretation of the PET data, the options to perform partialvolume correction and motion correction of PET data, and the opportunity to improve PET tracer uptake quantification. Not least, integrated PET/MR imaging protocols will provide information that would otherwise require several visits of the patient, thus increasing patient comfort. An improved diagnostic workflow algorithm could be established.

Several advantages can also be expected from a diagnostic perspective. PET/MR imaging may improve early diagnosis and prognosis by combining information on the potential risk for a neurodegenerative disorder and on the expected time to onset of cognitive problems in early stages of disease. With regard to differential diagnosis, combined assessment of PET and MR data may better exclude nonneurodegenerative pathologies and improve specific diagnosis by collecting accumulating evidence (i.e., information on causal neuropathology and on resulting neurodegenerative patterns). Furthermore, PET/MR imaging may allow excluding or identifying the presence of multiple pathologies. With regard to follow-up and therapy control, PET/MR imaging may allow a much better-informed quantification of disease progress or of therapy effects on several scales.

All these promising prospects lead to the expectation that the diagnostic assessment of dementia and other neurodegenerative disorders may clearly benefit from integrated PET/MR imaging, and that PET/MR imaging may be the method of choice for these indications. Preconditions include the elimination of some technologic limitations, access to the method and reimbursement, and the definition of intelligent diagnostic algorithms that define suitable and efficient combinations of imaging procedures for the individual diagnostic problem.

\section{DISCLOSURE}

Alexander Drzezga, Henryk Barthel, and Osama Sabri have been invited by Siemens Healthcare to present lectures on PET/MR imaging. Alexander Drzezga, Henryk Barthel, and Osama Sabri received speaker and consultant honoraria related to amyloid imaging from Bayer Healthcare and Piramal Imaging, and 
Alexander Drzezga received speaker and consultant honoraria from GE Healthcare and Avid/Lilly Pharmaceuticals. This work was also supported by grants from the Deutsche Forschungsgemeinschaft (DFG) and in part by the DFG, Großgeräteinitiative, which funded the installation of the PET/MR scanners at the Technische Universität München and at the University of Leipzig. No other potential conflict of interest relevant to this article was reported.

\section{REFERENCES}

1. Hebert LE, Weuve J, Scherr PA, Evans DA. Alzheimer disease in the United States (2010-2050) estimated using the 2010 census. Neurology. 2013;80:17781783.

2. Thies W, Bleiler L. 2013 Alzheimer's disease facts and figures. Alzheimers Dement. 2013;9:208-245.

3. Jack CR Jr, Wiste HJ, Weigand SD, et al. Amyloid-first and neurodegenerationfirst profiles characterize incident amyloid PET positivity. Neurology. 2013;81:1732-1740.

4. Jack CR Jr, Knopman DS, Jagust WJ, et al. Hypothetical model of dynamic biomarkers of the Alzheimer's pathological cascade. Lancet Neurol. 2010;9:119-128.

5. Scheltens PH. Structural neuroimaging of Alzheimer's disease and other dementias. Aging (Milano). 2001;13:203-209.

6. Chen Z, Zhong C. Decoding Alzheimer's disease from perturbed cerebral glucose metabolism: implications for diagnostic and therapeutic strategies. Prog Neurobiol. 2013;108:21-43.

7. Magistretti PJ, Pellerin L. Cellular mechanisms of brain energy metabolism and their relevance to functional brain imaging. Philos Trans $R$ Soc Lond B Biol Sci. 1999;354:1155-1163.

8. Barthel H, Gertz HJ, Dresel S, et al. Cerebral amyloid- $\beta$ PET with florbetaben $\left({ }^{18} \mathrm{~F}\right)$ in patients with Alzheimer's disease and healthy controls: a multicentre phase 2 diagnostic study. Lancet Neurol. 2011;10:424-435.

9. Clark CM, Pontecorvo MJ, Beach TG, et al. Cerebral PET with florbetapir compared with neuropathology at autopsy for detection of neuritic amyloid- $\beta$ plaques: a prospective cohort study. Lancet Neurol. 2012;11:669-678.

10. Landau SM, Thomas BA, Thurfjell L, et al. Amyloid PET imaging in Alzheimer's disease: a comparison of three radiotracers. Eur J Nucl Med Mol Imaging. March 20, 2014 [Epub ahead of print].

11. Jack CR Jr, Knopman DS, Jagust WJ, et al. Tracking pathophysiological processes in Alzheimer's disease: an updated hypothetical model of dynamic biomarkers. Lancet Neurol. 2013;12:207-216.

12. Bateman RJ, Xiong C, Benzinger TL, et al. Clinical and biomarker changes in dominantly inherited Alzheimer's disease. N Engl J Med. 2012;367:795-804.

13. Judenhofer MS, Wehrl HF, Newport DF, et al. Simultaneous PET-MRI: a new approach for functional and morphological imaging. Nat Med. 2008;14:459-465.

14. Schlemmer HP, Pichler BJ, Schmand M, et al. Simultaneous MR/PET imaging of the human brain: feasibility study. Radiology. 2008;248:1028-1035.

15. Drzezga A, Souvatzoglou M, Eiber M, et al. First clinical experience with integrated whole-body PET/MR: comparison to PET/CT in patients with oncologic diagnoses. J Nucl Med. 2012;53:845-855.

16. Eggers C, Szelies B, Bauer B, et al. Imaging of acetylcholine esterase activity in brainstem nuclei involved in regulation of sleep and wakefulness. Eur J Neurol. 2007;14:690-693.

17. Yanase D, Matsunari I, Yajima K, et al. Brain FDG PET study of normal aging in Japanese: effect of atrophy correction. Eur J Nucl Med Mol Imaging. 2005;32:794 805.

18. Sattler B, Jochimsen T, Barthel H, et al. Physical and organizational provision for installation, regulatory requirements and implementation of a simultaneous hybrid PET/MR-imaging system in an integrated research and clinical setting. MAGMA. 2013;26:159-171.

19. Chen J, Yao J, Thomasson D. Automatic determination of arterial input function for dynamic contrast enhanced MRI in tumor assessment. Med Image Comput Comput Assist Interv. 2008;11:594-601.

20. Chen K, Chen X, Renaut R, et al. Characterization of the image-derived carotid artery input function using independent component analysis for the quantitation of ${ }^{18} \mathrm{~F}$-fluorodeoxyglucose positron emission tomography images. Phys Med Biol. 2007;52:7055-7071.

21. Martinez-Möller A, Souvatzoglou M, Delso G, et al. Tissue classification as a potential approach for attenuation correction in whole-body PET/MRI: evaluation with PET/CT data. J Nucl Med. 2009;50:520-526.
22. Catana C, Benner T, van der Kouwe A, et al. MRI-assisted PET motion correction for neurologic studies in an integrated MR-PET scanner. J Nucl Med. 2011;52:154-161.

23. Musiek ES, Chen Y, Korczykowski M, et al. Direct comparison of fluorodeoxyglucose positron emission tomography and arterial spin labeling magnetic resonance imaging in Alzheimer's disease. Alzheimers Dement. 2012;8:51-59.

24. Andersen FL, Ladefoged CN, Beyer T, et al. Combined PET/MR imaging in neurology: MR-based attenuation correction implies a strong spatial bias when ignoring bone. Neuroimage. 2014;84:206-216.

25. Hitz S, Habekost C, Fürst S, et al. Systematic comparison of the performance of integrated whole-body PET/MR to conventional PET/CT for ${ }^{18} \mathrm{~F}$-FDG brain imaging in patients examined for suspected dementia. J Nucl Med. In press.

26. Braak E, Griffing K, Arai K, Bohl J, Bratzke H, Braak H. Neuropathology of Alzheimer's disease: what is new since A. Alzheimer? Eur Arch Psychiatry Clin Neurosci. 1999;249(suppl. 3):14-22.

27. Davies L, Wolska B, Hilbich C, et al. A4 amyloid protein deposition and the diagnosis of Alzheimer's disease: prevalence in aged brains determined by immunocytochemistry compared with conventional neuropathologic techniques. Neurology. 1988;38:1688-1693.

28. Dubois B, Feldman HH, Jacova C, et al. Research criteria for the diagnosis of Alzheimer's disease: revising the NINCDS-ADRDA criteria. Lancet Neurol. 2007;6:734-746.

29. Sperling RA, Aisen PS, Beckett LA, et al. Toward defining the preclinical stages of Alzheimer's disease: recommendations from the National Institute on AgingAlzheimer's Association workgroups on diagnostic guidelines for Alzheimer's disease. Alzheimers Dement. 2011;7:280-292.

30. Frisoni GB, Fox NC, Jack CR Jr, Scheltens P, Thompson PM. The clinical use of structural MRI in Alzheimer disease. Nat Rev Neurol. 2010;6:67-77.

31. Whitwell JL, Jack CR Jr. Comparisons between Alzheimer disease, frontotemporal lobar degeneration, and normal aging with brain mapping. Top Magn Reson Imaging. 2005;16:409-425.

32. Yuan Y, Gu ZX, Wei WS. Fluorodeoxyglucose-positron-emission tomography, single-photon emission tomography, and structural MR imaging for prediction of rapid conversion to Alzheimer disease in patients with mild cognitive impairment: a meta-analysis. AJNR Am J Neuroradiol. 2009;30:404-410.

33. Kawachi T, Ishii K, Sakamoto S, et al. Comparison of the diagnostic performance of FDG-PET and VBM-MRI in very mild Alzheimer's disease. Eur $J$ Nucl Med Mol Imaging. 2006;33:801-809.

34. Matsunari I, Samuraki M, Chen WP, et al. Comparison of ${ }^{18}$ F-FDG PET and optimized voxel-based morphometry for detection of Alzheimer's disease: aging effect on diagnostic performance. J Nucl Med. 2007;48:1961-1970.

35. Fleisher AS, Sun S, Taylor C, et al. Volumetric MRI vs clinical predictors of Alzheimer disease in mild cognitive impairment. Neurology. 2008;70:191-199.

36. Jack CR Jr, Vemuri P, Wiste HJ, et al. Evidence for ordering of Alzheimer disease biomarkers. Arch Neurol. 2011;68:1526-1535.

37. Salat DH, Buckner RL, Snyder AZ, et al. Thinning of the cerebral cortex in aging. Cereb Cortex. 2004;14:721-730.

38. Fox NC, Schott JM. Imaging cerebral atrophy: normal ageing to Alzheimer's disease. Lancet. 2004;363:392-394.

39. Hort J, O'Brien JT, Gainotti G, et al. EFNS guidelines for the diagnosis and management of Alzheimer's disease. Eur J Neurol. 2010;17:1236-1248.

40. Herholz K. FDG PET and differential diagnosis of dementia. Alzheimer Dis Assoc Disord. 1995;9:6-16.

41. Minoshima S, Foster NL, Sima AA, Frey KA, Albin RL, Kuhl DE. Alzheimer's disease versus dementia with Lewy bodies: cerebral metabolic distinction with autopsy confirmation. Ann Neurol. 2001;50:358-365.

42. Silverman DH, Small GW, Chang CY, et al. Positron emission tomography in evaluation of dementia: regional brain metabolism and long-term outcome. JAMA. 2001;286:2120-2127.

43. Knopman DS, Jack CR, Wiste HJ, et al. Short-term clinical outcomes for stages of NIA-AA preclinical Alzheimer disease. Neurology. 2012;78:1576-1582.

44. Klunk WE, Engler H, Nordberg A, et al. Imaging brain amyloid in Alzheimer's disease with Pittsburgh Compound-B. Ann Neurol. 2004;55:306-319.

45. Clark CM, Schneider JA, Bedell BJ, et al. Use of florbetapir-PET for imaging beta-amyloid pathology. JAMA. 2011;305:275-283.

46. Vandenberghe R, Van Laere K, Ivanoiu A, et al. ${ }^{18}$ F-flutemetamol amyloid imaging in Alzheimer disease and mild cognitive impairment: a phase 2 trial. Ann Neurol. 2010;68:319-329.

47. Mintun MA, Larossa GN, Sheline YI, et al. ${ }^{11} \mathrm{C}$-PIB in a nondemented population: potential antecedent marker of Alzheimer disease. Neurology. 2006;67:446452.

48. Pike KE, Savage G, Villemagne VL, et al. Beta-amyloid imaging and memory in non-demented individuals: evidence for preclinical Alzheimer's disease. Brain. 2007;130:2837-2844. 
49. Villemagne VL, Pike KE, Darby D, et al. Abeta deposits in older non-demented individuals with cognitive decline are indicative of preclinical Alzheimer's disease. Neuropsychologia. 2008;46:1688-1697.

50. Koivunen J, Scheinin N, Virta JR, et al. Amyloid PET imaging in patients with mild cognitive impairment: a 2-year follow-up study. Neurology. 2011;76:1085-1090.

51. Okello A, Koivunen J, Edison P, et al. Conversion of amyloid positive and negative MCI to AD over 3 years: an ${ }^{11}$ C-PIB PET study. Neurology. 2009;73:754-760.

52. Nordberg A, Carter SF, Rinne J, et al. A European multicentre PET study of fibrillar amyloid in Alzheimer's disease. Eur J Nucl Med Mol Imaging. 2013;40:104-114.

53. Salloway S, Sperling R, Fox NC, et al. Two phase 3 trials of bapineuzumab in mild-to-moderate Alzheimer's disease. N Engl J Med. 2014;370:322-333.

54. Donaghy P, Thomas AJ, O'Brien JT. Amyloid PET imaging in Lewy body disorders. Am J Geriatr Psychiatry. July 3, 2013 [Epub ahead of print].

55. Wolk DA, Price JC, Madeira C, et al. Amyloid imaging in dementias with atypical presentation. Alzheimers Dement. 2012;8:389-398.

56. Rabinovici GD, Rosen HJ, Alkalay A, et al. Amyloid vs FDG-PET in the differential diagnosis of AD and FTLD. Neurology. 2011;77:2034-2042.

57. Rinne JO, Brooks DJ, Rossor MN, et al. ${ }^{11} \mathrm{C}$-PiB PET assessment of change in fibrillar amyloid-beta load in patients with Alzheimer's disease treated with bapineuzumab: a phase 2, double-blind, placebo-controlled, ascending-dose study. Lancet Neurol. 2010;9:363-372.

58. Perneczky R, Drzezga A, Diehl-Schmid J, et al. Schooling mediates brain reserve in Alzheimer's disease: findings of fluoro-deoxy-glucose-positron emission tomography. J Neurol Neurosurg Psychiatry. 2006;77:1060-1063.

59. McKhann GM, Knopman DS, Chertkow H, et al. The diagnosis of dementia due to Alzheimer's disease: recommendations from the National Institute on AgingAlzheimer's Association workgroups on diagnostic guidelines for Alzheimer's disease. Alzheimers Dement. 2011;7:263-269.

60. Jack CR Jr, Lowe VJ, Senjem ML, et al. ${ }^{11} \mathrm{C}-\mathrm{PiB}$ and structural MRI provide complementary information in imaging of Alzheimer's disease and amnestic mild cognitive impairment. Brain. 2008;131:665-680.
61. Frisoni GB, Bocchetta M, Chételat G, et al. Imaging markers for Alzheimer disease: which vs how. Neurology. 2013;81:487-500.

62. Brück A, Virta JR, Koivunen J, et al. ${ }^{11} \mathrm{C}-\mathrm{PIB},{ }^{18} \mathrm{~F}-\mathrm{FDG}$ and MR imaging in patients with mild cognitive impairment. Eur J Nucl Med Mol Imaging. 2013;40:1567-1572.

63. Hatashita S, Yamasaki H. Diagnosed mild cognitive impairment due to Alzheimer's disease with PET biomarkers of beta amyloid and neuronal dysfunction. PLOS ONE. 2013;8:e66877.

64. Zhang D, Shen D. Predicting future clinical changes of MCI patients using longitudinal and multimodal biomarkers. PLOS ONE. 2012;7:e33182.

65. Jack CR Jr, Wiste HJ, Vemuri P, et al. Brain beta-amyloid measures and magnetic resonance imaging atrophy both predict time-to-progression from mild cognitive impairment to Alzheimer's disease. Brain. 2010;133:3336-3348.

66. Albert MS, DeKosky ST, Dickson D, et al. The diagnosis of mild cognitive impairment due to Alzheimer's disease: recommendations from the National Institute on Aging-Alzheimer's Association workgroups on diagnostic guidelines for Alzheimer's disease. Alzheimers Dement. 2011;7:270-279.

67. Knopman DS, Jack CR Jr, Wiste HJ, et al. Brain injury biomarkers are not dependent on $\beta$-amyloid in normal elderly. Ann Neurol. 2013;73:472-480.

68. Dukart J, Mueller K, Horstmann A, et al. Combined evaluation of FDG-PET and MRI improves detection and differentiation of dementia. PLOS ONE. 2011;6: e18111.

69. Cha YH, Jog MA, Kim YC, Chakrapani S, Kraman SM, Wang DJ. Regional correlation between resting state FDG PET and pCASL perfusion MRI. J Cereb Blood Flow Metab. 2013;33:1909-1914.

70. Meyer PT, Hellwig S, Amtage F, et al. Dual-biomarker imaging of regional cerebral amyloid load and neuronal activity in dementia with PET and ${ }^{11} \mathrm{C}$ labeled Pittsburgh compound B. J Nucl Med. 2011;52:393-400.

71. Rostomian AH, Madison C, Rabinovici GD, Jagust WJ. Early ${ }^{11} \mathrm{C}$-PIB frames and ${ }^{18}$ F-FDG PET measures are comparable: a study validated in a cohort of $\mathrm{AD}$ and FTLD patients. $J$ Nucl Med. 2011;52:173-179. 